\title{
Exact solution of equations for proton localization in neutron star matter
}

\author{
Sebastian Kubis* and Włodzimierz Wójcik \\ Institute of Physics, Cracow University of Technology, Podchorażych 1, 30-084 Kraków, Poland \\ (Received 1 June 2015; revised manuscript received 17 September 2015; published 3 November 2015)
}

\begin{abstract}
The rigorous treatment of proton localization phenomenon in asymmetric nuclear matter is presented. The solution of proton wave function and neutron background distribution is found by the use of the extended Thomas-Fermi approach. The minimum of energy is obtained in the Wigner-Seitz approximation of a spherically symmetric cell. The analysis of four different nuclear models suggests that the proton localization is likely to take place in the interior of a neutron star.
\end{abstract}

DOI: 10.1103/PhysRevC.92.055801

PACS number(s): 21.65.Cd, 21.65.Mn, 26.60.-c

\section{INTRODUCTION}

The interior of a neutron star contains the densest forms of matter in the Universe. The central density is as high as 5 to 10 times the nuclear equilibrium density $n_{0}=0.16 \mathrm{fm}^{-3}$. Most of the mass of the star is placed in its liquid core covered by a thin crust ( $<1 \mathrm{~km}$ for typical neutron star) whose bottom edge is located at around $0.5 n_{0}$. Above this density the matter is well described by the Fermi liquid-a mixture of nucleons and leptons. In comparison to the matter present inside the stable nuclei, the matter in a neutron star is highly asymmetric as a consequence of the $\beta$ equilibrium taking place between nucleons and leptons. It is convenient to express the asymmetry by the proton fraction $x=n_{p} / n$, where $n_{p}, n$ are the proton and baryon number density. The proton fraction is between 0.4 and 0.5 in nuclei, whereas in neutron star matter at $n_{0}$ it is equal to $4 \%$ what is exactly determined by the saturation point properties. The proton abundance at higher density is not well known and different nuclear interactions models lead to very large discrepancies in the $x(n)$ behavior. There are models which predict that $x$ does not exceed $10 \%$ in a full range of densities. When the proton fraction is not high, protons can be treated as the small admixture to the neutron background, where direct proton-proton interaction is negligible and hence protons can be regarded as impurities in the neutron matter. Therefore, a description of this system by single proton in neutron background is justified. The attractive nature of the proton-neutron interaction may result in an instability of homogeneously distributed protons [1-3]. In the paper [3] the polaron behavior of a proton impurity in dense neutron matter was discussed. A single proton in neutron matter can lower its energy by inducing the density inhomogeneity around it. Instead of forming the Fermi sea, protons occupy the ground state with zero momentum above some critical density. It occurs when the localized proton with properly distorted neutron background has smaller energy than the system with the proton described by the plane waves. Such a state of matter has intriguing magnetic properties that have been shown in [1,6-8]. It exhibits, e.g., a crystallization of proton impurities in the neutron star interior $[9,10]$ and affects the cooling process of neutron stars [11,12]. In the

*skubis@pk.edu.pl general discussion it is important to distinguish between proton localization and other kinds of instabilities. In the exotic matter, like kaon condensation [13], hyperonic and quark matter [14], when the phase transition occurs, it is likely that pasta phases are formed. However in normal nucleon-lepton matter at high density such a phenomenon was not observed.

In the papers [2,3] the proton localization has been analyzed in a simplified manner. A variational approach to a cell containing one proton was proposed. However, the minimization of the energy was achieved with respect to Gaussian-type trial function with only one parameter for both proton wave function and neutron background. Moreover, the cell was treated as a system with infinite volume $V \rightarrow \infty$ which means that the method is applicable to a very small ( $x$ smaller than $1 \%$ ) proton fraction.

The aim of this work is to solve exactly the Lagrange-Euler equations corresponding to the variational approach proposed in the original works. We also abandon the assumption of infinitely large cell. This means we may take into account higher proton fractions and thus, extend the class of nuclear models in the analysis.

The equation of state of supranuclear density in the neutron star core cannot be calculated unambiguously $[19,20]$. Instead, there are many theoretical models with many different components. From a theoretical point of view the hyperon presence above density $\sim 3 n_{0}$ seems to be very likely, however the most typical for hyperon - soft equation of state-leads to a rather low maximum mass of a neutron star, what is in contradiction with the recent massive pulsars observations $[15,16]$. The reconciliation of hyperons with those mass limits is a matter of hot debate [17]. In the context of proton localization the hyperon presence would be especially interesting. Typically, the $\Lambda$ hyperon production in dense matter simultaneously diminishes the proton abundance [18] and could support, in this manner, the proton localization. The other hyperons with low abundances could also be candidates for the localization. The hyperon localization would be of particular interest as the localization usually blocks the conventional neutrino processes (both direct and modified URCA) and would change drastically the cooling story of the neutron star.

In this work, however, we limit ourselves to the pure nucleonic matter as first we are going to examine in more rigorous detail the past ideas and avoid a complication coming from the inclusion the exotic components. We choose four 


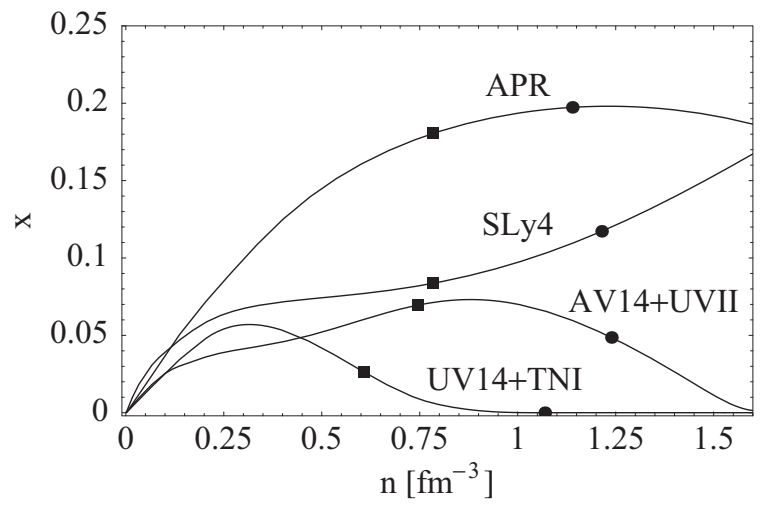

FIG. 1. The proton fraction for different models used in the calculation: APR, SLy4, AV14+UVII and UV14+TNI. The squares indicate the proton localization threshold and the full dots indicate the central density of a star with maximum mass.

representatives of EoS for nucleonic matter. Two of them, APR (in original work called A18+ $\delta v+\mathrm{UIX}^{*}$ ) [21] and SLy4 [22], are very common in the description of neutron star structure. However one must remember that SLy4 is based on the low energy expansion of the Skyrme potential and extrapolation to high density is doubtful. We decided to include two other models AV14+UVII and UV14+TNI [23]. Although being out of date, they were used in previous calculations for proton localization and it was important to compare our present results with the past ones. The inclusion of the last model (UV14+TNI) is the most dubious as it does not fulfill the $2 M_{\odot}[15,16]$ criterion for maximum neutron star mass, however it is interesting, because of complete vanishing of proton fraction at density around $5 n_{0}$. In Fig. 1 the proton fraction in $\beta$-equilibrated matter is shown for the four selected nuclear models.

The present paper is organized as follows. A short review of the variational method for a finite-size Wigner-Seitz cell is presented in Sec. II. In Sec. III the numerical method for solving the equations is explained. The results are shown and discussed for various nuclear models in Sec. IV.

\section{A PROTON IN NEUTRON BACKGROUND}

In order to calculate the energy of nuclear matter with localized protons we treat the proton as a quantum particle described by its wave function $\Psi(\boldsymbol{r})$ whereas the neutrons are represented by a density distribution function $n_{n}(\boldsymbol{r})$. As in the work [3] we assume that one proton occupies a spherical Wigner-Seitz (W-S) cell filled with a large number of neutrons. Neutrons are treated in the local density approximation according to differential Thomas-Fermi scheme [24]. The energy of the cell is expressed by the integral over the whole cell volume $V_{W S}=1 / \bar{n}_{p}$,

$E\left[\psi, n_{n}\right]=\int_{V_{W S}}\left[\Psi^{*}\left(-\frac{\nabla^{2}}{2 m_{p}}+\mu_{p}\right) \Psi+\varepsilon+B_{N}\left(\nabla n_{n}\right)^{2}\right] d^{3} r$.

The nuclear matter energy density $\varepsilon$ is the thermodynamical function which depends directly on nucleon densities $\varepsilon\left(n_{n}, n_{p}\right)$.
Its functional form is completely determined by the adopted nuclear model. The chemical potentials are defined as usual:

$$
\mu_{p}=\left(\frac{\partial \varepsilon}{\partial n_{p}}\right) n_{n}, \quad \mu_{n}=\left(\frac{\partial \varepsilon}{\partial n_{n}}\right) n_{p} .
$$

In the energy functional Eq. (1) the energy density $\varepsilon$ and the proton chemical potential $\mu_{p}$ get the space dependence by the local neutron density: $\varepsilon(r)=\varepsilon\left(n_{n}(r), 0\right)$ and in the same way $\mu_{p}(r)=\mu_{p}\left(n_{n}(r), 0\right)$. The constant coefficient $B_{N}$ describes the gradient contribution and it is fitted to the surface properties of nuclei, here we adopt the value $B_{N}=31.6 \mathrm{MeV} \mathrm{fm}^{5}$ [2]. The W-S cell radius is given by the proton density for homogeneous system $R_{W S}=\left(3 / 4 \pi \bar{n}_{p}\right)^{1 / 3}$, where $\bar{n}_{p}=x n$ and $n$ is the mean baryon number.

The cell energy should be minimized under constraints of fixed proton and neutron number:

$$
\begin{aligned}
\int_{V_{W S}} \Psi^{*} \Psi d^{3} r & =1, \\
\int_{V_{W S}} n_{n} d^{3} r & =V_{W S} \bar{n}_{n},
\end{aligned}
$$

where $\bar{n}_{n}=(1-x) n$ is the mean neutron number in the case of the homogeneous system. The constraints expressed by Eqs. (3), (4) require the following Lagrange multipliers $\lambda_{p}, \lambda_{n}$ :

$$
\tilde{E}=E-\lambda_{p} \int\left(\Psi^{*} \Psi-1 / V_{W S}\right) d^{3} r-\lambda_{n} \int\left(n_{n}-\bar{n}_{n}\right) d^{3} r .
$$

For the isolated, spherically symmetric W-S cell we impose the following boundary conditions:

$$
\begin{array}{ll}
\frac{\partial \Psi}{\partial r}(0)=0 & \Psi\left(R_{W S}\right)=0, \\
\frac{\partial n_{n}}{\partial r}(0)=0 & \frac{\partial n_{n}}{\partial r}\left(R_{W S}\right)=0 .
\end{array}
$$

From the Lagrange-Euler equations for the minimum of $\tilde{E}$ one may remark that the Lagrange multipliers $\lambda_{p}$ and $\lambda_{n}$ correspond to the physical quantities such as the eigenvalue $E_{p}$ of the proton wave function and the neutron chemical potential $\mu_{n}$ at the cell boundary:

$$
\lambda_{p}=E_{p}, \quad \lambda_{n}=\left.\mu_{n}\right|_{R_{W S}},
$$

and then, finally, one may write the Lagrange-Euler equations in the form

$$
\begin{aligned}
\frac{-\nabla^{2}}{2 m_{p}} \Psi+\mu_{p} \Psi & =E_{p} \Psi, \\
\frac{\partial \mu_{p}}{\partial n_{n}} \Psi^{*} \Psi+\Delta \mu_{n}-2 B_{N} \nabla^{2} n_{n} & =0,
\end{aligned}
$$

where $\Delta \mu_{n}=\mu_{n}\left(n_{n}(r), 0\right)-\left.\mu_{n}\right|_{R_{W S}}$ is the difference between the local chemical potential and its boundary value. The first equation (8) represents the Schrödinger equation for the spherically symmetric proton wave function $\Psi(r)$ with the eigenvalue $E_{p}$. The coupling to neutron density $n_{n}(r)$ comes from the chemical potential $\mu_{p}\left(n_{n}, 0\right)$. The second equation (9) is the nonlinear elliptic equation for the neutron density distribution $n_{n}(r)$ coupled to the proton density $\Psi^{*} \Psi$. 
The proton localization occurs if at a given mean density $n$ there exists a proton wave function with a negative eigenvalue $E_{p}<0$ and when the energy of the homogeneous system of nucleons is greater than the energy $E\left[\Psi, n_{n}\right]$ of a system with distorted densities, that means

$$
\Delta E=E\left[\Psi, n_{n}\right]-\varepsilon(n(1-x), n x) V_{W S}<0 .
$$

In this way, by solving Eqs. (8), (9), we obtain a family of solutions parametrized with the mean density of matter $n$.

\section{THE METHOD}

The mean baryon density $n$ does not enter directly to Eqs. (8), (9). The average density $n$ is determined indirectly by the second constraint, Eq. (4). Therefore, in numerical solving, it is simpler to set the value at the boundary

$$
\left.n_{n}^{\infty} \equiv n_{n}\right|_{R_{W S}},
$$

find the proton function and neutron background, and then finally derive the mean density from the relation

$$
n=\frac{1}{V_{W S}}\left(1+\int_{V_{W S}} n_{n}(r) d^{3} r\right) .
$$

The set of Eqs. (8), (9) was solved by the relaxation method explained in the following.

As an initial approximation, the Gaussian-type function was taken for the proton wave function and for the neutron density. In the $i$ th step, the iteration had two stages: in the first we found the ground state solution $\Psi^{(i+1)}, E_{p}^{(i+1)}$ of the Schrödinger equation including $n_{n}^{(i)}$. In the second step we solve Eq. (9) for

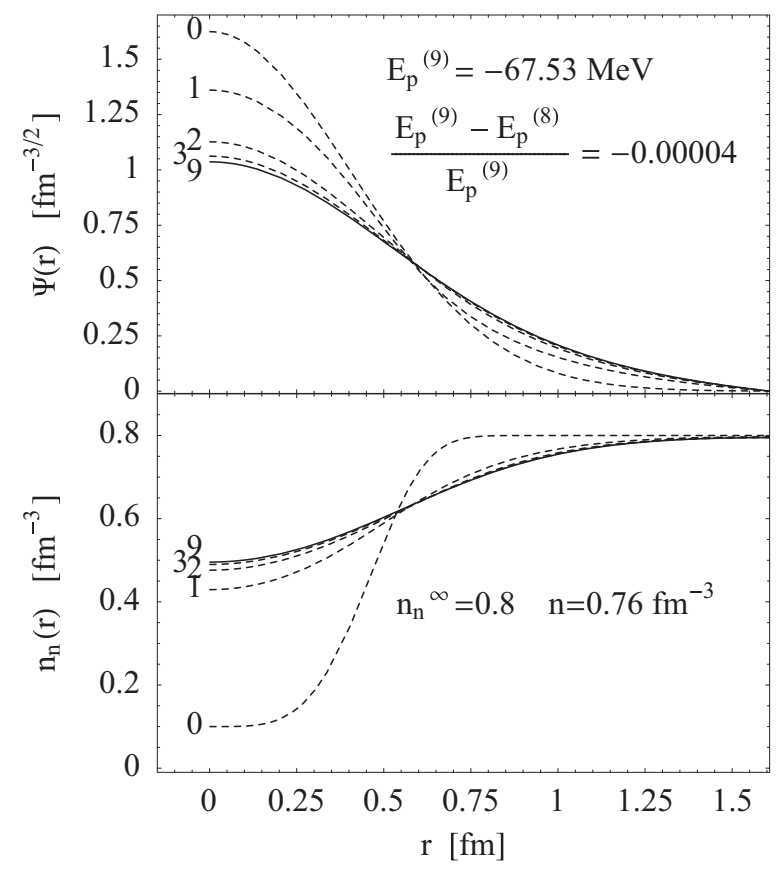

FIG. 2. The subsequent steps in the relaxation method for proton wave function $\psi(r)$ (upper panel) and neutron density $n_{n}(r)$ (lower panel) in the nuclear model AV14+UVII. The solid curve represents the final results after 9 steps when the accuracy equal to $10^{-4}$ was achieved for ground energy $E_{p}$. $n_{n}^{(i+1)}$ including $\Psi^{(i+1)}$. The iteration was continued up to the point where the eigenvalue does not change more than a given accuracy. The procedure appeared to converge quickly, usually the accuracy equal to $10^{-4}$ was achieved in not more than 15 steps. Figure 2 represents the convergence of the iteration for the chosen density $n_{n}^{\infty}=0.8$ in the AV14+UVII model.

\section{RESULTS}

The proton localization scheme described in the previous sections was then analyzed for the accepted nuclear models: APR, SLy4, AV14+UVII, and UV14+TNI. For all of them the proton localization occurred. Comparing the localization threshold obtained here with the results of previous works based on the approximate variational method with Gaussian proton profile (see Table I in [5]), one observes systematically lower values resulting from the present method. A large change in the proton radius was obtained as well. In our calculation it is always much smaller. The comparison of our results with those of [5] is presented in Table I.

As a conclusion one may say that the correction to the threshold density is of the order of $10 \%$ whereas for the proton radius reaches $30 \%$. It seems natural that the finite size of the W-S cell makes the proton radius smaller. The new values of localization threshold $n_{n}^{\infty}$ are also smaller since in our calculations both proton wave function and neutron background present exact solutions of assumed equations.

The three models (APR, SLy4, AV14+UVII) were analyzed in the whole range of available density: from the threshold for localization (see first row for the particular model in Table II) to the maximum density which is determined by the maximum neutron star mass (the last row the particular model). In the case of the fourth one (UV14+TNI), the range of density relevant for localization was between the threshold and the point where the protons disappear that means $x=0$. It occurs for $n=$ $1.07 \mathrm{fm}^{-3}$. In Fig. 3 the evolution with the baryon density of proton wave function and neutron background distribution is shown. The vertical lines indicate the W-S cell radius $R_{W S}$. For the APR model the $R_{W S}$ takes the smallest values, around $1 \mathrm{fm}$ which means that the cell contains about five neutrons. Such a small number of neutrons questions the local density approximation in the case of the APR model. However for the rest of the models, the $R_{W S}$ is greater and W-S cell contains from 10 to several hundred of neutrons which justifies the description of neutrons by its local density $n_{n}(r)$.

The behavior of the proton wave function and neutron density is similar as in the previous approximate calculations [2-4]. The proton mean radius $\left\langle r_{p}\right\rangle$ decreases

TABLE I. Comparison of present results (new) with work [5] (old) for the threshold density and proton mean radius.

\begin{tabular}{lccccc}
\hline \hline & \multicolumn{2}{c}{$n_{n}^{\infty}\left[\mathrm{fm}^{-3}\right]$} & & \multicolumn{2}{c}{$\left\langle r_{p}\right\rangle[\mathrm{fm}]$} \\
\cline { 2 - 3 } \cline { 6 - 7 } & old & new & & old & new \\
\hline APR & 0.819 & 0.784 & & 0.878 & 0.562 \\
AV14+UVII & 0.789 & 0.745 & & 0.971 & 0.747 \\
UV14+TNI & 0.731 & 0.610 & & 1.209 & 1.023 \\
\hline \hline
\end{tabular}


TABLE II. Various parameters above the threshold on the proton localization.

\begin{tabular}{|c|c|c|c|c|}
\hline$n_{n}^{\infty}\left[\mathrm{fm}^{-3}\right]$ & $n\left[\mathrm{fm}^{-3}\right]$ & $\Delta E / A[\mathrm{MeV}]$ & $E_{p}[\mathrm{MeV}]$ & $\left\langle r_{p}\right\rangle[\mathrm{fm}]$ \\
\hline \multicolumn{5}{|c|}{ APR } \\
\hline 0.784 & 0.854 & 0 & -44.5 & 0.562 \\
\hline 0.846 & 0.924 & -5.23 & -88.5 & 0.529 \\
\hline 0.907 & 0.994 & -11.42 & -140.6 & 0.499 \\
\hline 0.969 & 1.065 & -18.89 & -201.0 & 0.471 \\
\hline 1.030 & 1.135 & -27.61 & -269.8 & 0.446 \\
\hline \multicolumn{5}{|c|}{ SLy4 } \\
\hline 0.785 & 0.805 & 0 & -45.9 & 0.674 \\
\hline 0.879 & 0.905 & -1.55 & -84.6 & 0.606 \\
\hline 0.972 & 1.007 & -3.73 & -131.2 & 0.548 \\
\hline 1.066 & 1.110 & -6.75 & -185.0 & 0.500 \\
\hline 1.160 & 1.214 & -10.81 & -245.6 & 0.459 \\
\hline \multicolumn{5}{|c|}{ AV14+UVII } \\
\hline 0.745 & 0.763 & 0 & -34.0 & 0.747 \\
\hline 0.861 & 0.885 & -3.52 & -114.5 & 0.624 \\
\hline 0.978 & 1.005 & -8.41 & -230.1 & 0.528 \\
\hline 1.094 & 1.123 & -13.44 & -376.1 & 0.456 \\
\hline 1.210 & 1.239 & -16.98 & -550.7 & 0.404 \\
\hline \multicolumn{5}{|c|}{ UV14+TNI } \\
\hline 0.610 & 0.608 & 0 & -23.6 & 1.023 \\
\hline 0.725 & 0.723 & -0.22 & -71.8 & 0.826 \\
\hline 0.840 & 0.838 & -0.50 & -137.2 & 0.660 \\
\hline 0.955 & 0.954 & -0.80 & -213.0 & 0.564 \\
\hline 1.070 & 1.069 & -1.08 & -297.0 & 0.500 \\
\hline
\end{tabular}

whereas the depth of the neutron well increases with the mean density of matter. The particular values of quantities relevant for the proton localization are shown in Table II. The first two columns present the neutron density $n_{n}^{\infty}$ at the W-S boundary Eq. (11) and the mean baryon density $n$. The $\Delta E / A$ is the energy difference between the homogeneous matter and the state with localized proton Eq. (10) taken per total number of baryons in the cell. The $E_{p}$ presents the proton energy eigenvalue. For all models the localization energy $\Delta E / A$ increases with the density and the same happens to the proton energy $E_{p}$, so one may conclude the proton is stronger localized at higher densities. An interesting fact is that, in the case of the UV14+TNI model, although the proton fraction is very small, the strength of localization, measured by the energy difference $\Delta E / A$ takes the smallest values in comparison to the other models.

\section{SUMMARY}

In the present work we have solved the Lagrange-Euler equations for a proton impurity with the extended ThomasFermi approach for neutron background. The proton was treated as quantum particle immersed in the quasiclassical neutron sea. In a previous work the proton abundance was assumed to be infinitely small, i.e., the Wigner-Seitz cell was infinitely large, $R_{W S} \rightarrow \infty$. Here we kept finite $R_{W S}$ determined by the proton fraction which is fixed by the $\beta$-equilibrium occurring in neutron star matter. By minimizing the energy in the finite-size Wigner-Seitz cell we found an
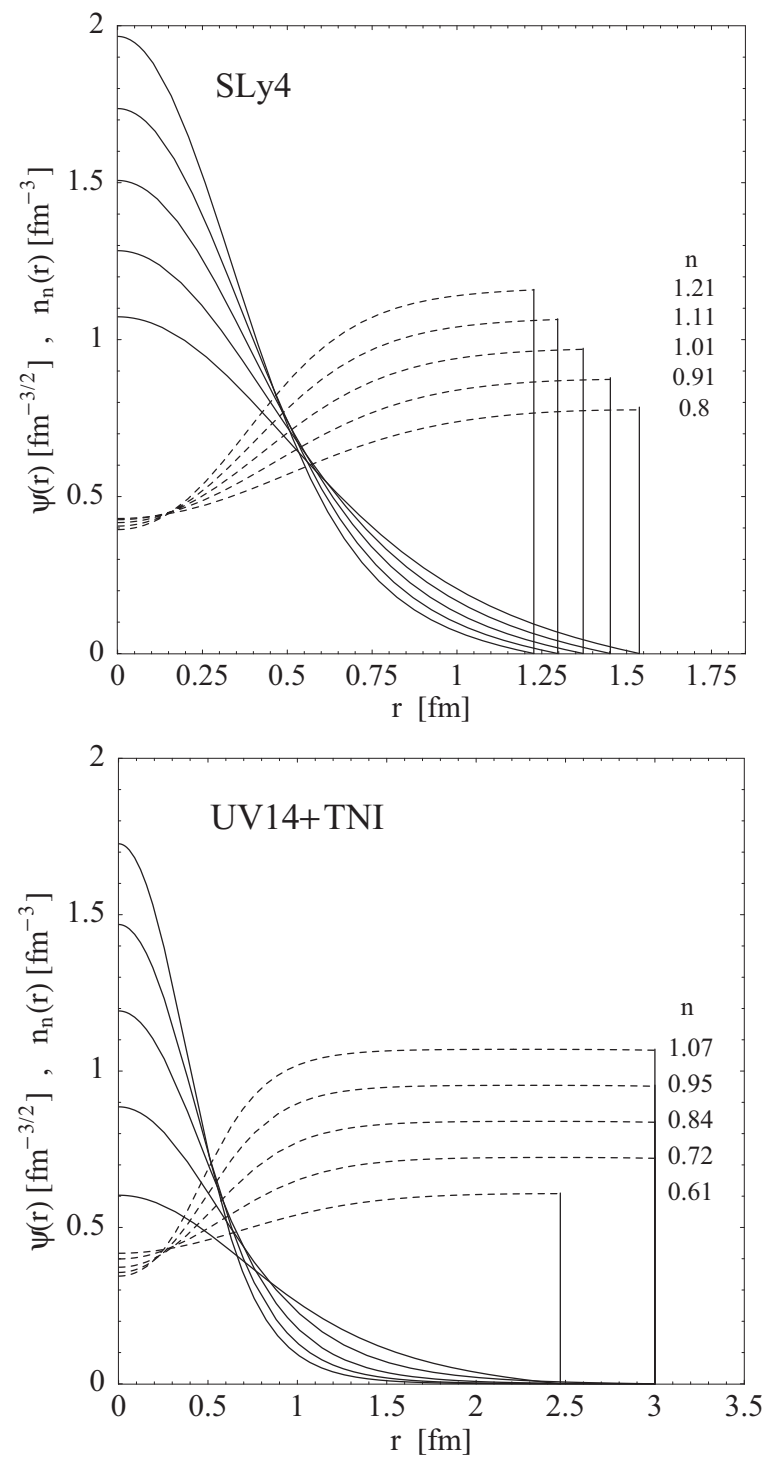

FIG. 3. The evolution of proton wave function (solid) and neutron background (dashed) with increasing mean baryon density $n$ for the two nuclear models. Vertical lines indicate the position of $R_{W S}$. In the case of UV14+TNI, the distribution is truncated because for densities above $0.7 \mathrm{fm}^{-3}$ the $R_{W S}$ was greater than $3 \mathrm{fm}^{-3}$.

exact solution for the proton wave function and neutron background. It turn out that proton localization still occurs for all the presented models. The localization threshold is slightly lower than in the previous work where the one-parameter method for energy minimization was used [4]. In this work we have investigated, in a rigorous way, the earlier ideas of the proton localization and have shown the phenomenon is plausible and worth further research. A required improvement would be to perform the calculation in the $\mathrm{W}-\mathrm{S}$ cell with periodic boundary conditions. This issue was attacked in Ref. [9] however in a very simplified manner. A desired modification of the approach would be the inclusion of hyperonic degrees of freedom, especially that both hyperons presence and proton localization influence the neutron star cooling $[11,12]$. 


\section{ACKNOWLEDGMENTS}

We are grateful to Marek Kutschera and Adam Szmagliński for helpful feedback at the early stage of this work and fruitful discussions. We wish also to thank the referee for questions that have helped us to improve the clarity of our presentation. This work was done in the framework of statutory activities.
[1] M. Kutschera and W. Wójcik, Phys. Lett. B 223, 11 (1989).

[2] M. Kutschera and W. Wójcik, Acta Phys. Polon. B 21, 823 (1990).

[3] M. Kutschera and W. Wójcik, Phys. Rev. C 47, 1077 (1993).

[4] M. Kutschera, S. Stachniewicz, A. Szmagliński, and W. Wójcik, Acta Phys. Polon. B 33, 743 (2002).

[5] A. Szmagliński, W. Wójcik, and M. Kutschera, Acta Phys. Polon. B 37, 277 (2006).

[6] M. Kutschera and W. Wójcik, Acta Phys. Polon. B 23, 947 (1992).

[7] M. Kutschera and W. Wójcik, Phys. Lett. B 325, 271 (1994).

[8] M. Kutschera and W. Wójcik, Acta Phys. Polon. A 92, 375 (1997).

[9] M. Kutschera and W. Wójcik, Nucl. Phys A 581, 706 (1995).

[10] A. Y. Potekhin, Phys. Usp. 53, 1235 (2010).

[11] D. A. Baiko and P. Haensel, Acta Phys. Polon. B 30, 1097 (1999).

[12] D. A. Baiko and P. Haensel, Astron. Astrophys. 356, 171 (2000).

[13] T. Maruyama, T. Tatsumi, D. N. Voskresensky, T. Tanigawa, T. Endo, and S. Chiba, Phys. Rev. C 73, 035802 (2006).
[14] T. Maruyama, S. Chiba, H. J. Schulze, and T. Tatsumi, Phys. Rev. D 76, 123015 (2007).

[15] P. B. Demorest, T. Pennucci, S. M. Ransom, M. S. E. Roberts, and J. W. T. Hessels, Nature 467, 1081 (2010).

[16] J. Antoniadis et al., Science 340, 6131 (2013).

[17] I. Vidaña, AIP Conf. Proc. 1645, 79 (2015).

[18] J. Schaffner-Bielich, Nucl. Phys. A 835, 279 (2010).

[19] J. M. Lattimer and M. Prakash, Phys. Rep. 442, 109 (2007).

[20] P. Haensel, A. Y. Potekhin, and D. G. Yakovlev, Neutron Stars 1: Equation of State and Structure (Springer, Berlin, 2006).

[21] A. Akmal, V. R. Pandharipande, and D. G. Ravenhall, Phys. Rev. C 58, 1804 (1998).

[22] E. Chabanat, P. Bonche, P. Haensel, J. Meyer, and R. Schaeffer, Nucl. Phys. A 635, 231 (1998).

[23] R. B. Wiringa, V. Fiks, and A. Fabrocini, Phys. Rev. C 38, 1010 (1988).

[24] G. Baym, H. A. Bethe, and C. Pethick, Nucl. Phys. A 175, 225 (1971). 